\title{
INFLUENCIAS ERGONÓMICAS EN LA VISUALIZACIÓN DE LA INFORMACIÓN
}

\section{INFLUÊNCIAS ERGONÔMICAS NA VISUALIZAÇÃO DA INFORMAÇÃO}

\author{
Deborah Torres Ponjuan - eborahponjuan@infomed.sld.cu \\ Doutoranda em Documentação e Informação Científica. \\ Docente da Universidade de Havana.
}

\section{Gloria Ponjuan Dante - gponjuan@infomed.sld.cu Doutora em Ciências da Informação. Professora da Universidade de Havana.}

\begin{abstract}
Resumen
Este trabajo pretende analizar y esclarecer algunas aproximaciones concernientes a la relación interdisciplinar que se establece entre la Ergonomía Cognitiva y la Visualización de la Información, en la búsqueda de un reconocimiento de la necesidad de considerar criterios ergonómicos en el diseño y evaluación de las interfaces visuales de la información por los profesionales de la información. La interpretación y complementación del enfoque histórico y epistemológico se basó en el análisis cualitativo de un conjunto de fuentes de información proveniente de literatura científica relevante sobre ambos campos.
\end{abstract}

\section{Palabras clave}

Ergonomía Cognitiva - Visualización de la información. Ergonomía Cognitiva - Interfaces Visuales.

\section{INTRODUCCIÓN}

La utilización de propuestas teóricas sobre las relaciones entre disciplinas para analizar las distintas influencias dentro de un área científica de naturaleza interdisciplinar, contribuye a una mejor interpretación de su comportamiento y a la adecuada utilización de distintos métodos y herramientas. La Visualización de la Información, desde su surgimiento, ha sido objeto de investigación por distintas comunidades científicas, con mayor fuerza desde la Ciencia de la Computación y las Ciencias Cognitivas, que han aportado con sus concepciones, métodos y técnicas a una evolución fundamentalmente orientadas a la praxis. No obstante, a pesar de su fuerte influencia tecnológica y cognitiva 
no suele considerarse en el estudio de su identidad como disciplina científica, las contribuciones que en particular la Ergonomía Cognitiva ha realizado, a partir de las bases evolutivas de ambos campos.

Este artículo pretende analizar y establecer cómo ocurre la relación entre ambas desde sus inicios con una visión integradora, y contribuir al conocimiento de investigadores y profesionales al brindar una comprensión más profunda de cómo considerar el enfoque ergonómico en la Visualización de la Información.

\section{ERGONOMÍA COGNITIVA Y VISUALIZACIÓN DE LA INFORMACIÓN: BASES HISTÓRICAS}

La preocupación cambiante por la interacción del ser humano con la naturaleza, los objetos y la sociedad en el marco de las actividades productivas, ha estado siempre pendiente de las transformaciones históricas de la naturaleza del trabajo.

En el entorno de la sociedad industrial y del Positivismo, en la segunda mitad del siglo XIX, el científico polaco Jastrzebowski (1857) introduce las bases de la Ergonomía al estudiar la actividad humana con un enfoque marcadamente filosófico. En su propuesta, divide el trabajo en dos categorías: el trabajo "útil", destinado a mejorar las cosas comunes y a las personas, y el trabajo "dañino" o deshonroso, que conduce al deterioro. En particular considera que el trabajo útil necesita de fuerzas motoras, de la razón (pensamiento y razonamiento) y espirituales, y lo clasifica en trabajo físico, estético, racional y moral (KARWOWSKI, 2005).

En los inicios del siglo XX, con la Segunda Revolución Industrial, la ciencia comienza a ser un elemento crucial del progreso económico, social y un ingrediente inevitable de las tecnologías. La búsqueda de la eficiencia y las exigencias de la mecanización extensa de los sistemas de producción estimularon la investigación de psicólogos y filósofos, entre otros. Es de destacar las contribuciones de Frederick Winslow Taylor, que introduce el estudio "científico" del trabajo (TAYLOR, 1911).

La II Guerra Mundial despierta el interés intenso de la Psicología por probar las potencialidades humanas como resultado del empleo de complejos equipos, reorientando las metas investigativas posteriores hacia la reducción del tiempo de adiestramiento, eliminar errores y aumentar el ritmo del comportamiento experto. La resolución de problemas se convierte entonces en un elemento importante en la búsqueda de la eficiencia en los procesos. Es entonces que comienza a dominar la perspectiva clásica de 
la Ergonomía relacionada con la calidad al trabajar y se concentra en los "aspectos físicos del trabajo y las capacidades humanas" (HOLLNAGEL, 1997). En 1949, el psicólogo británico Murrell (EDHOLM; MURRELL, 1974) une los términos ergon (del griego, trabajo) y nomía (del griego, norma, trabajo), define a la Ergonomía ${ }^{1}$ como "el estudio de la relación entre el hombre y su ambiente de trabajo", y la considera como una ciencia aplicada, tecnológica, o ambas, dedicada al estudio de los sistemas de trabajo con una aproximación centrada en el humano (KARWOWSKI, 2005).

El proceso de amplificación de la ciencia y la tecnología en el desarrollo industrial condujo a un aumento de las capacidades humanas para realizar el trabajo físico, y a un interés por el estudio de las mismas, lo que conllevó a que a partir de la segunda mitad del siglo XX, de acuerdo al estudio de Helander (1997), la Ergonomía se fuera adaptando a las cambiantes necesidades de la sociedad (Tabla 1), revelando la sensibilidad de su investigación por los factores contextuales como los cambios culturales o tecnológicos, y en los últimos años por los acercamientos sociales.

\begin{tabular}{|c|c|}
\hline Décadas & Alcance \\
\hline$' 50$ & Ergonomía militar \\
\hline$' 60$ & Ergonomía industrial \\
\hline$' 70$ & Ergonomía del consumidor \\
\hline 80 & $\begin{array}{c}\text { Ergonomía de la interacción } \\
\text { humano-computadora y del } \\
\text { software }\end{array}$ \\
\hline '90 & $\begin{array}{c}\text { Ergonomía cognitiva y } \\
\text { organizacional }\end{array}$ \\
\hline 2000 & $\begin{array}{c}\text { Comunicación global y eco- } \\
\text { ergonomía }\end{array}$ \\
\hline
\end{tabular}

Tabla 1 - Evolución histórica de la ergonomía, según Helander (1997)

En las décadas del '50 y al '60 se desarrollaron numerosas teorías sobre el tratamiento de la información, como por ejemplo, la Cibernética y la Teoría de la Información, que junto al uso creciente de las máquinas para el tratamiento de la información propiciaron en estas disciplinas los estudios ergonómicos. En particular, en el

\footnotetext{
${ }^{1}$ Por razones históricas, el término Ergonomía se ha utilizado con el mismo sentido que el término Factores Humanos. La misma disciplina fue nombrada posteriormente Ergonomía en Europa y Factores Humanos en EE.UU. Actualmente los dos términos son utilizados indistintamente.
} 
entorno de la Computación, los cambios tecnológicos y de operación atrajeron la mayor atención hacia la innovación en el hardware y al desarrollo de software más versátil, y condujeron a que los usuarios prácticos pusieran más interés en lo que hacían con las computadoras que en cómo lo hacían. Como consecuencia, en 1965, la International Business Machines Corporation (IBM) introduce la Computación en el campo de los negocios, y comienza a incrementarse el volumen de publicaciones científicas en ese campo con una orientación hacia el sujeto como objeto de estudio (GRUDIN, 2005).

El surgimiento y evolución de las Ciencias Cognitivas en la segunda mitad del siglo $X X$ y el impacto de los continuos cambios tecnológicos condujeron a un encuentro entre la Ergonomía y la Psicología Cognitiva, y al reconocimiento de una nueva perspectiva en la Ergonomía, relacionada con la calidad del trabajo y orientada a los aspectos psicológicos del trabajo (cómo la mente afecta al trabajo y cómo el trabajo afecta a la mente) pero no a comprender la naturaleza de la cognición humana), imponiendo la emergencia de la Ergonomía Cognitiva. Esta combinación refleja un cambio de paradigma en la Psicología: del conductismo al cognitivismo, es decir, "de la manera en que actuamos a la manera que pensamos" (HOLLNAGEL, 2001).

A partir de los años '70, el desarrollo de la Ergonomía Cognitiva estuvo marcada por los rápidos adelantos tecnológicos, económicos e informacionales. Su interés por estudiar el desarrollo de las interfaces como mediadoras destinadas a facilitar la interacción se incrementa a partir de este momento debido a tres factores fundamentales: la rápida evolución de la tecnología en las computadoras, que introdujo mejoras (en la velocidad, la capacidad de memoria y la resolución de las pantallas) que posibilitaron el desarrollo de las interfaces; la diversidad de usuarios de los sistemas de computadora (con edades, sexo, idioma, cultura, entrenamiento, experiencia, habilidades, motivaciones e intereses diferentes, que demandan interfaces más flexibles y adaptables para un amplio rango de necesidades y expectativas); y la intensa competencia del mercado, que obliga a los productores de software a reducir costos y mantener productos distintivos destinados a mejorar la productividad de los usuarios, con una mayor calidad y menores costos de entrenamiento.

En 1970, se fundan el Human Sciences and Advanced Technology (HUSAT) en la Universidad de Loughborough en Gran Bretaña, para el desarrollo de investigaciones ergonómicas con énfasis en la Human Computer Interaction $(\mathrm{HCl})$; y el Xerox Palo Alto Research Center (PARC) en EE.UU., para impulsar la tecnología computacional y desarrollar nuevos hardware, lenguajes de programación y ambientes de programación. 
HUSAT se centró en la investigación sobre Ergonomía y consideró los Factores Humanos como factores organizacionales. Por su parte, los investigadores de PARC extendieron los Factores Humanos hacia la "cognición de alto nivel" (GRUDIN, 2005).

Por otra parte, el auge de los sistemas de información geográfica (Geographic information systems, GIS) con base en la cartografía tradicional, influye en las nuevas concepciones del diseño de interfaces que posteriormente se concibe en el uso de metáforas visuales con un enfoque geográfico en la Visualización de la información. (BÖRNER; CHEN; BOYACK, 2003)

A mediados de los '70 y en los '80, el interés por los modelos matemáticos en los procesos de recuperación de información se desplaza hacia las representaciones que conllevan procesos mentales complejos, interactivos y contextuales; se supone que las estructuras de conocimiento cambian gracias a la información, y se comienzan a identificar los procesos mentales con los programas informáticos (HERNÁNDEZ, 2006). Se comienza a estudiar especialmente los aspectos cualitativos de la interacción en la recuperación de la información por varias disciplinas $(\mathrm{HCl}$, Inteligencia Artificial, Ciencia Cognitiva, Ciencia de la computación, entre otras), con el fin de facilitar el intercambio de información y la interacción entre ordenadores y personas.

A inicios de la década del '80, la IBM incorpora a varios psicólogos cognitivos en sus grupos de investigación, la mayoría familiarizados con el software interactivo pero no con la literatura investigativa de los factores humanos. A la vez, la revista Communications of the ACM abrió el departamento "Human Aspects of Computing", y el Special Interest Group on Social and Behavioral Science Computing (SIGSOC) de la Association for Computing Machinery (ACM) amplió su alcance en 1981 para cubrir el diseño y uso de software interactivo, moviéndose hacia el último enfoque en 1982 y adoptando el nombre de Computer-Human Interaction (SIGCHI). Aunque en 1983 su conferencia (CHI 83) fue coauspiciada por la Human Factors Society, esto no vuelve a suceder. Las dudas acerca de la investigación sobre los Factores Humanos se evidencian en "The Psychology of Human-Computer Interaction" que introdujo la modelación cognitiva y se orientó hacia el comportamiento experto y la reforma de los factores humanos (CARD et al., 1983, apud GRUDIN, 2005).

Desde la segunda mitad de los '80 el modelado de la mente humana como un sistema de procesamiento de información pierde relevancia, cambio que se le atribuye a la Ergonomía Cognitiva. (HOLLNAGEL, 2001). La HCl, por su parte, enfatiza en la ciencia 
de la Computación y se reduce el énfasis en la ciencia cognitiva, aparece entonces la "ingeniería cognitiva" y la "ingeniería de usabilidad", y se evitan los temas sobre factores humanos, desapareciendo el interés por los mismos. En $\mathrm{CHI}$ se aborda en esta década un enfoque "centrado en el usuario" y el diseño interactivo basado en prototipos (GRUDIN, 2005). Su evolución se refleja en las influyentes contribuciones de Norman, un científico cognitivo que acuñó el término "ingeniería cognitiva" y que definió las funciones de satisfacción del usuario basado en la rapidez de uso, facilidad de aprendizaje, conocimiento requerido y errores (NORMAN, 1983).

En paralelo, desde la IBM T. J. Watson Research Center, se genera una diversidad de enfoques entre sus investigadores: en 1984, su grupo de Factores Humanos se debilita y aparece el User Interface Institute; Gould se mantuvo en los Factores Humanos y actuó como presidente de la Human Factors Society entre 1987-88; mientras Brooks, Curtis, Green, Shneiderman y otros fundadores de $\mathrm{CHI}$ continuaron las investigaciones sobre la psicología de la programación (GRUDIN, 2005), rama que dio origen a la Visualización de Información a principios de los '90.

A partir de 1985, la investigación sobre la interacción entre personas y computadoras es influenciada por el desarrollo de los sistemas de información, la extensión del uso de Internet e Intranet, el desarrollo de las interfaces gráficas con el usuario (Graphical User Interface, GUI) y la World Wide Web; así como por el rol del gobierno como usuario y partidario de la investigación y el desarrollo del campo de los Factores Humanos y la Ergonomía Cognitiva.

En octubre de 1986 la National Science Foundation (NSF) de EE.UU. asigna máxima prioridad al desarrollo de herramientas visuales, incluyendo hardware, software y herramientas visuales para gráficos y técnicas de procesamiento de imágenes, y "lanza una importante iniciativa interdisciplinaria en la Visualización Científica", con el fin de mostrar cómo la computadora puede servir como intermediaria en el proceso de la asimilación rápida de la información (CARD; MACKINLAY; SHNEIDERMAN, 1999). McCormick, DeFanti y Brown (1987), en su informe para la NSF, introducen el concepto de "visualización en el dominio científico" y surge entonces la Visualización Científica como una disciplina especial.

La Visualización de Información como disciplina comienza a gestarse a finales de los años '80, impulsada por el Human-Computer Interaction Laboratory (HCIL) de la Universidad de Maryland y el Xerox Palo Alto Research Center (Xerox PARC), y motivada 
fundamentalmente por el interés de aplicar las experiencias de la Visualización Científica en otras nuevas que permitieran tratar con datos más complejos, con grandes volúmenes de documentos y nuevas tipologías documentales. Card, Mackinlay, Shneiderman (1999) afirman que el término "Visualización de Información" fue usado por primera vez por Robertson, Card y Mackinlay (1989), para referirse a una propuesta de arquitectura cognitiva para interfaces de usuario interactivas. Los autores la consideraron análoga a la Visualización Científica, y en ella proponían el uso de objetos animados bidimensionales (2D) y tridimensionales (3D) para representar la información y sus relaciones estructurales. Consideraba pues el estudio de elementos del diseño de las interfaces de los sistemas de computadora que afectan las actividades humanas (capacidades y limitaciones) en su interacción, relacionadas además con el conocimiento y el procesamiento de la información con fuertes influencias ergonómicas.

\section{ALCANCES DE LA ERGONOMÍA COGNITIVA}

La International Ergonomics Association (IEA) define a la Ergonomía Cognitiva como "el área de la Ergonomía que estudia los procesos mentales, tales como percepción, cognición, memoria, razonamiento y emoción, y cómo afectan las interacciones entre las personas y los productos, los sistemas, y los ambientes. Sus tópicos de estudio más relevantes incluyen: carga de trabajo mental, cognición, toma de decisiones, desempeño experto, interacción humano-computadora, fiabilidad humana, estrés de trabajo, entrenamiento, diferencias culturales, actitudes, placer y motivación."²

Incluye pues el estudio de elementos del diseño de las interfaces de los sistemas de computadora que afectan las actividades humanas (capacidades y limitaciones) relacionadas con el conocimiento y el procesamiento de la información en el entorno de trabajo, el trabajo cooperativo, la modelación de usuarios y sistemas, el aprendizaje y el diseño de sistemas.

En relación con la $\mathrm{HCl}$, la perspectiva de la Ergonomía Cognitiva se resume en la manera en que se presenta la información: "presentar la información correcta, en la forma correcta y en el tiempo correcto" (HOLLNAGEL, 1997). Tres de sus tópicos fundamentales de estudio: la usabilidad, los criterios ergonómicos y los métodos de evaluación de la usabilidad, prestan especial atención a los componentes conceptuales principales del

\footnotetext{
2 Tomada de: INTERNATIONAL ERGONOMICS ASSOCIATION (2003) IEA Triennial Report 2000-2003. Disponible en: http://www.iea.cc/browse.php?contID=what_is_ergonomics [Consultado: 23/5/2007].
} 
dominio de la $\mathrm{HCl}$ (participantes, interacción, interfaz, propósito, tareas, medioambiente) y las relaciones entre ellos (Storrs, 1989).

La aplicación de las nociones de la Ergonomía Cognitiva en los estudios de las interfaces de usuario, se han orientado fundamentalmente hacia la modelación de los procesos perceptivos y cognitivos $^{3}$, y a garantizar la usabilidad a través de criterios específicos para el diseño, la evaluación, y la prevención y corrección de errores.

El modelado cognitivo de los usuarios ayuda a obtener, a través de la comprensión de los procesos cognitivos propios del usuario, diferencias entre los comportamientos de las personas con diferentes estilos de aprendizaje y a diseñar sistemas más cercanos a la perspectiva del usuario (WILSON, 1999).

Los criterios ergonómicos son extremadamente útiles para definir qué cualidades se deben atribuir al sistema para que satisfagan al usuario en su utilización. Se basan en una filosofía centrada en el usuario, lo que implica considerar aspectos perceptivos, cognitivos, socio-culturales, psíquicos, experticidad y dominio del equipamiento del usuario, así como aspectos formales y de contenido en la representación. Se destacan particularmente los de Bastien y Scapin (1993), quienes diseñaron criterios ergonómicos con la finalidad de incorporar consideraciones desde la ergonomía al desarrollo y evaluación de las interfaces. Estos autores aclaran que sus criterios están destinados a evaluar la "calidad ergonómica de los sistemas interactivos", término que cubre la usabilidad en su sentido más amplio: calidad de uso, hasta el punto en que los usuarios puedan alcanzar fácilmente los objetivos de la interacción, y la utilidad, entendida como la posibilidad de alcanzar los objetivos a través de los aspectos funcionales de la interacción.

Los criterios ergonómicos constituyen "un puente entre las necesidades y tareas de los usuarios por un lado, y el diseño de la interfaz usuario por la otra" (NIGAY; VERNIER, 1998); apuntando pues a dos mundos: el estudio del usuario y el diseño de la interfaz por el otro. Se deben tener en cuenta los criterios de ambas partes, tanto las características del usuario y de sus tareas, como sus implicaciones en las reglas del diseño.

\footnotetext{
${ }^{3}$ Un modelo cognitivo es "una representación del conocimiento de una persona" compuesta por un conjunto de elementos conceptuales, las relaciones entre ellos y las relaciones entre grupos de elementos asociados (GILLAN y BREEDIN, 1990).
} 


\section{ENFOQUES ERGONÓMICOS EN LA VISUALIZACIÓN DE INFORMACIÓN}

La Visualización de la Información investiga el diseño de las representaciones visuales interactivas, apoyadas usualmente por herramientas informáticas, que supuestamente permitan una rápida asimilación y comprensión de un volumen grande de información, buscando optimizar la carga cognitiva en la representación visual de las estructuras, relaciones y patrones de información, y brindar nuevas visiones analíticas diferentes a partir de los datos contenidos en la información que se representa.

Sus propósitos fundamentales se han relacionado con acciones como descubrir, comprender, comunicar y educar, aunque en los últimos años éstos se han sintetizado en dos: descubrir y explicar. Un descubrimiento asociado a la resolución de novedosos problemas, a la identificación de fenómenos desconocidos, y a la comprensión, como otra forma de descubrimiento; y el uso explicativo de la visualización, con funciones comunicativas y educacionales (HOOK, 2007). Esto es posible a partir de las posibilidades de revelar estructuras emergentes dentro de los datos (patrones contenidos en la información), e interactuar y explorar de forma intuitiva, ampliando la perspectiva del usuario sobre las relaciones que se establecen entre los elementos asociados a la temática que está estudiando.

Distintos autores han abordado sus investigaciones desde la Psicología y la Ergonomía Cognitiva para analizar la Visualización. Algunos ejemplos relevantes son los siguientes:

- Csinger (1992), quien elabora un modelo de visualización a partir de la Psicología.

- Ingram y Benford (1995), quienes proponen soluciones para lograr la legibilidad desde el diseño de la Visualización de Información para mejorar la navegabilidad.

- Brath (1997a), quien brinda una guía para herramientas tridimensionales de Visualización de Información, tanto desde la perspectiva de la interacción como de la visualización, e insiste en la necesidad de garantizar la calidad de los datos y su correspondencia con el objetivo.

- Bartram (1997), quien analiza las propiedades perceptivas e interpretativas del movimiento en la Visualización de Información. 
- Card, Mackinlay, y Shneiderman (1999), quienes proponen "seis vías a partir de las cuales las visualizaciones aumentan la cognición: 1) incrementando la memoria y procesando los recursos disponibles de los usuarios; 2) reduciendo la búsqueda de información; 3) usando representaciones visuales para reforzar el descubrimiento de patrones; 4) habilitando operaciones de inferencia perceptivas; 5) usando mecanismos perceptivos de atención para el monitoreo y 6) codificando la información en un medio manipulable".

- Crapo et al. (2000), quienes analizan el proceso de modelación en la Visualización desde un punto de vista cognitivo.

- Kraidy (2002) quien analiza el impacto cognitivo de la Visualización de información en los medios digitales y en la Educación.

Desde una mirada ergonómica, la Visualización de Información utiliza las representaciones gráficas como apoyo para suplir las limitaciones de la memoria de trabajo, transforma lo abstracto (conceptos y relaciones lógicas, temporales, sintácticas) en concreto (objetos y relaciones visuales espaciales) para facilitar el razonamiento. El carácter concreto de la imagen induce al razonamiento a elegir una opción (o mantener una estrategia de discriminar vías de trabajo alternativas que se tratarán sucesivamente) y su carácter espacial obliga a una explicitud en las representaciones visuales, lo que puede favorecer el rápido acceso y la comprensión de la información, de personas con distintos niveles de conocimientos previos y experticia.

La indización espacial en la imagen permite una rápida localización y acceso a los datos, y por tanto permite agilizar el razonamiento. Utiliza distintas formas de representación visual de la información, en la búsqueda de una comprensión más profunda y extensa de las relaciones implicadas entre los elementos contenidos y representados en la información y de sus relaciones intertextuales con otros conceptos y representaciones. Permite, en definitiva, crear nuevas relaciones mentales a partir de las existentes y potenciar las inferencias.

La comprensión efectiva de la información visualizada puede ser compleja, depende de la percepción, cognición, de las tareas específicas de los usuarios y de sus metas (TORY; MÖLLER, 2004) y puede estar influida incluso por factores físicos (iluminación, entorno, etc.) o psicológicos (el usuario puede tener prisa o desviar su atención sobre algún detalle importante de la representación visual por priorizar otras tareas). Por esta razón, la Ergonomía cognitiva se interesa por este campo y es necesario considerar sus 
concepciones fundamentales en el diseño y evaluación de las herramientas de Visualización de Información con la finalidad de facilitar la interacción del usuario con la información y aprovechar al máximo las potencialidades que se brindan.

En gran medida las herramientas de la Visualización de Información dependen de su usabilidad, por lo que además de prestar la atención acostumbrada a los algoritmos de visualización por los diseñadores, se recomienda determinar previamente las guías, requerimientos funcionales y restricciones del sistema sobre la base del estudio de los usuarios y sus limitaciones, preferencias y valores, objetivos (generales y específicos), conocimiento y experiencia, procesos de pensamiento, ambiente físico, tareas que debe realizar, problemas que el sistema debería resolver y otras tareas a ser ejecutadas en función de la carga cognitiva del usuario, información que permite además disponer de determinados criterios ergonómicos para evaluar las distintas etapas de desarrollo de la visualización. No obstante, aunque existe una gran variedad de modelos y técnicas asociadas a la Visualización de la Información, no existe aún un consenso sobre la evaluación de su usabilidad.

Para examinar la funcionalidad y facilidad de los sistemas de visualización, se mencionan en general los estudios de usuario (KOSARA et al., 2003), las inspecciones de usabilidad (TORY; MÖLLER, 2005) y la comparación empírica de técnicas (CHEN; YU, 2000). Algunos de estos métodos requieren de técnicas de validación analítica (heurística o por inspección) que verifican la ergonomía de la interfaz, buscando identificar posibles problemas en la interacción del usuario con la computadora.

Fundamentalmente, la evaluación de las herramientas de Visualización de Información se aborda desde cuatro áreas temáticas:

- Experimentos controlados, que miden el desempeño del usuario y comparan elementos del diseño (WISS; CARR; JONSSON, 1998; JUAREZ; HENDRICKSON; GARRETT, 2000; CHEN; YU, 2000; CHEN; CZERWINSKI, 2000; KOBSA, 2004; PLAISANT; GROSJEAN; BEDERSON, 2002; WANG; TEOH; MA, 2006).

- Evaluación de usabilidad de una herramienta, que proporcionan criterios de los usuarios que aporten a los diseñadores cómo perfeccionar el diseño. Como métodos incluyen a la evaluación con usuarios (KOBSA, 2004; FREITAS et. al., 2002), la evaluación heurística (BALDONADO; WOODRUFF; KUCHINSKY, 2000; AMAR; STASKO, 2004; ZUK; CARPENDALE, 2006; TORY; MÖLLER, 
2005) y la evaluación métrica (BRATH, 1997a; WINCKLER; PALANQUE; FREITAS, 2004; PILLAT; VALIATI; FREITAS, 2005).

- Estudios de caso de herramientas en escenarios reales (el menos común), con la ventaja de demostrar la factibilidad en el contexto de los usuarios, y la desventaja de no ser "replicables ni generalizables". Incluyen como métodos los estudios longitudinales o estudios de campo (GONZÁLEZ; KOBSA, 2003; PLAISANT, 2004; SHNEIDERMAN; PLAISANT, 2006), y la evaluación basada en la percepción (SARAIYA; NORTH, DUNCA, 2004, 2005).

- Uso de modelos cognitivos (Juarez-Espinosa, 2003).

Distintos autores han propuesto además criterios ergonómicos factibles para evaluar la efectividad de la representación de las herramientas de Visualización de Información (BRATH, 1997b; FREITAS et al., 2002; LUZZARDI; FREITAS, 2004; PLAISANT, 2004). EI análisis de las distintas variantes, revela una serie de recomendaciones generales para la evaluación de tales herramientas, como las que se resumen a continuación:

1. La evaluación debe estar presente en todas las etapas del desarrollo de las herramientas, y apoyarse y ser controlada por principios heurísticos y criterios ergonómicos particulares que cubran las necesidades de la Visualización de Información.

2. La usabilidad se debe considerar como calidad de uso, con el apoyo de distintas taxonomías definidas.

3. No se debe obviar la percepción del usuario participante en la evaluación, especialmente ante representaciones complejas, considerando distintos niveles de complejidad en la evaluación de las tareas, prestando especial atención a la arquitectura de la información y a la conducta cognitiva del usuario ante la información, intentando para ello un acercamiento a situaciones reales.

4. Se deben escoger en los experimentos controlados, usuarios con distintos niveles de conocimiento, experiencia y habilidades, asignando en cada caso un juego diferente de tareas adecuado a sus características, en busca de resultados más realistas, prestando especial atención a los procesos de transformación de los datos y a las técnicas escogidas para ello.

5. Se deben utilizar distintos métodos y contextos, y contrastar resultados.

6. Considerar en la evaluación ayudas alternativas y facilidades de aprendizaje de la herramienta en cuestión. 
7. Utilizar bases de conocimiento que apoyen el proceso de evaluación, y registrar los resultados para investigaciones futuras.

\section{IMPORTANCIA PARA LOS PROFESIONALES DE LA INFORMACIÓN}

Los profesionales de la información han encontrado interesante la aplicación de las herramientas de la Visualización de la Información fundamentalmente para explorar nuevas alternativas en los estudios métricos (SMALL, 1999), en la representación y análisis de espacios documentales y dominios de conocimiento (BÖRNER; CHEN; BOYACK, 2003), en la minería de datos y de textos (KEIM; KRIEGEL, 1996; KEIM, 2002), en la recuperación de información (KOSHMAN, 2004, 2006), así como propuestas de visualización para bibliotecas digitales (BÖRNER, 2000), OPAC's (FRÍAS; TRAVIESO RODRíGUEZ, 2003), entre otros.

Según CHEN (2005) algunos de los principales problemas de la Visualización de Información asociados al reto del enfoque centrado en el usuario se relacionan con el desarrollo de metodologías orientadas a garantizar la usabilidad, la identificación de las principales tareas perceptivo-cognitivas en la recuperación de la información, disponer de un conocimiento previo del dominio de aplicación que permita adaptar los sistemas al conocimiento acumulado de sus usuarios y reduzca la dependencia de expertos; desarrollar acciones y productos de educación y entrenamiento que acerquen las herramientas a sus potenciales beneficiarios; elaborar métricas de calidad propias, especialmente para su evaluación y selección; estudiar la escalabilidad y la estética para mejorar los algoritmos actuales; desarrollar técnicas que le faciliten al usuario interactuar con los datos y explorarlos visualmente, analizarlos para probar hipótesis; así como visualizar dominios de conocimiento, a partir de la capacidad de la Visualización de Información de mostrar una gran cantidad de información e interactuar con ella para facilitar la representación de aspectos relevantes de distintas áreas de conocimiento.

La mayoría de estos problemas puede enfrentarse con estudios desde la Ciencia de la Información, la que no ha consolidado aún un pensamiento teórico sobre este tema en particular por la complejidad de las distintas perspectivas interdisciplinares, la existencia de múltiples herramientas cuya factibilidad de aplicación en el área necesitan ser probadas y la fuerte dependencia tecnológica, estadística y matemática en sus bases teóricas. 
Asociar métodos y técnicas de visualización a las necesidades y problemas específicos de usuarios con distintas capacidades y niveles de formación; investigar qué herramientas de visualización son más efectivas para apoyar la gestión de la información y el conocimiento sobre la base de técnicas, métodos y criterios de evaluación adecuados de los procesos que se realizan; lograr la comprensión acerca de cómo los distintos grupos de usuarios perciben los resultados que se logran al aplicar las distintas técnicas de visualización, y estar en condiciones de proponer criterios a tener en cuenta en soluciones futuras, son retos que los profesionales de la información deben enfrentar desde perspectivas ergonómicas e interdisciplinares.

\section{CONCLUSIONES}

La Ergonomía Cognitiva tiene sus raíces en el encuentro entre la Psicología Cognitiva y la Ergonomía en la segunda mitad del siglo XX, producto del surgimiento y evolución de las Ciencias cognitivas y los acelerados cambios tecnológicos que, con impacto en distintas ciencias, se produjeron. Extendió su influencia hacia el dominio de la $\mathrm{HCl}$ gracias a importantes iniciativas académicas multidisciplinares desarrolladas por instituciones privadas como la IBM y la Xerox PARC, que apoyaban la aplicación de la ciencia cognitiva a la Computación. La Visualización de la Información, por su parte, emerge en los 90 como una nueva disciplina producto también de una integración disciplinar con un fuerte enfoque cognitivo, producto de una integración disciplinar que parte de la aplicación de la psicología a la programación.

La evolución de ambas disciplinas, con raíces comunes, vuelve a coincidir en el interés de la Ergonomía Cognitiva por el diseño y evaluación de las herramientas de la Visualización de la Información, dirigido hacia los estudios de las interfaces de usuario, la modelación de los procesos perceptivos y cognitivos, y a garantizar la usabilidad a través de criterios específicos.

Las posibilidades que brindan distintas herramientas de Visualización de Información de transformar la vista de la imagen representada partiendo de un panorama global en la representación y filtrar o enfocarse en algún detalle, entre otras, permite indagaciones o profundizaciones progresivas, y proporcionar sobre los mismos datos de partida la posibilidad de múltiples representaciones para apoyar el razonamiento.

Los profesionales de la información han incorporado el uso de herramientas de Visualización de Información en su ámbito profesional con múltiples propósitos: 
representar gráficamente datos de un determinado dominio de aplicación, descubrir relaciones, tendencias y fenómenos interesantes, interpretar y comprender la información representada a partir de las relaciones espaciales exhibidas, deducir nuevos conocimientos, tomar decisiones validando o refutando hipótesis, o explicar presentando la información a otros. Es importante pues considerar las nociones ergonómicas en los métodos de evaluación particular de cada herramienta, con la finalidad de lograr una interacción más fácil con la información visualizada, y mejorar los criterios de selección de herramientas más eficaces.

\section{REFERÊNCIAS}

AMAR, R.; STASKO, J. A Knowledge Task-Based Framework for Design and Evaluation of Information Visualizations. Proceedings of IEEE InfoVis, p. 143-9, 2004.

BALDONADO, M. Q. W.; WOODRUFF, A.; KUCHINSKY, A. Guidelines for Using Multiple Views in Information Visualization. Proceedings of AVI '00, ACM Press, New York, USA, p. 110-19, 2000.

BARTRAM, L. Perceptual and Interpretative Properties of Motion for Information Visualization. Proceedings of the 1997 workshop on New paradigms in information visualization and manipulation (NPIV '97), p. 3-7, 1997.

BASTIEN, J.M.C.; Scapin, D. L. Ergonomic criteria for the evaluation of human-computer interfaces (Technical report No. 156), Rocquencourt, France: Institut National de Recherché en Informatique et en Automatique (INRIA), 1993.

BÖRNER, K. Visible Threads: A smart VR interface to digital libraries. Proceedings of IST/SPIE's 12th Annual International Symposium: Electronic Imaging 2000, Visual Data Exploration and Analysis (SPIE 2000), San Jose, CA, p. 228-237.

BÖRNER; CHEN; BOYACK. Visualizing Knowledge Domains. Annual Review of Information Science and Technology (ARIST), v. 37, p. 186, 2003.

BRATH, R. Metrics for Effective Information Visualization. Proceedings for IEEE Symposium on Information Visualization, v. 20-1, p.108-11, 126, 1997 a.

BRATH, R. 3D Interactive Information Visualization: Guidelines from experience and analysis of applications. Proceedings of the Seventh International Conference on HumanComputer Interaction ( $\mathrm{HCl}$ International '97), San Francisco, California, USA, August 2429, 1997, Volumen 2, Elsevier, 1997b. Disponible en: www.oculusinfo.com/papers/HClintl1.pdf.

CARD, S.; MACKINLAY, J.; SHNEIDERMAN, B. (Eds.). Readings in Information Visualization: using vision to think. San Francisco: Morgan Kaufmann, 1999. 
CHEN, C. Top 10 Unsolved Information Visualization Problems. Computer Graphics and Applications, IEEE, v. 25, n. 4, p. 12-6, 2005.

CHEN, C.; CZERWINSKI, M. Empirical evaluation of information visualization: an introduction. International Journal of Human-Computer Studies. v. 53, p. 631-5, 2000.

CHEN, C.; YU, Y. Empirical Studies of Information Visualization: A Meta-Analysis. International Journal of Human-Computer Studies, v. 53, p. 851-66, 2000.

CRAPO, A. W. et al. Visualization and the process of modeling: a cognitive-theoretic view. Proceedings of the Sixth ACM SIGKDD international Conference on Knowledge Discovery and Data Mining (KDD '00). ACM Press, New York, p. 218-26, 2000.

CSINGER, A. The Psychology of Visualization. Technical Report. UMI Order Number: TR92-28. University of British Columbia, 1992. Disponible en:

http://www2.iath.virginia.edu/time/readings/visualization-representation/psychology -ofvisualization.pdf.

EDHOLM, O.G.; MURRELL, K.F.H. The Ergonomics Society: A History, 1949 - 1970. Ergonomics, Suppl., London: Taylor \& Francis, (1974)

FREITAS, C. M. D. S. et al. Evaluating Usability of Information Visualization Techniques. Proceedings of Advanced Visual Interfaces (AVI '02), 2002. Disponible en:

www.inf.ufrgs.br/cg/publications/carla/FreitasEtAl-IHC.pdf. Acesso em: 14 abr. 2007.

FRÍAS, J. A.; TRAVIESO RODRÍGUEZ, C. La visualización de la información bibliográfica en los catálogos en línea y en entorno web: tendencias de investigación. In:

Tendencias de investigación en organización del conocimiento. Salamanca: Ediciones Universidad de Salamanca, 2003, p. 455-61.

GILLAN, D. J.; Breedin, S. D. Designers' models of the human-computer interface.

Proceedings of the SIGCHI Conference on Human Factors in Computing Systems:

Empowering People. J. C. Chew and J. Whiteside, Eds. CHI '90. ACM Press, New York, NY, p. 391-8, 1990.

GONZÁLEZ, V.; KOBSA, A. A Workplace Study of the Adoption of Information Visualization Systems. 3rd International Conference on Knowledge Management (IKNOW'03), Graz, Austria, 2003.

GRUDIN, J. Three Faces of Human-Computer Interaction. IEEE Annals of the History of Computing, v. 27, n. 4, p. 46-62, 2005.

HELANDER, M.G. Forty years of the IEA: some reflections on the evolution of ergonomics. Ergonomics, v. 40, n. 10, p. 952-61, 1997.

HERNÁNDEZ Organización y representación del conocimiento: paradigmas, hipertextos y fundación metamodélica. Tesis para optar por el grado de Doctor en Ciencias de la Información. Universidad de La Habana. Facultad de Comunicación, 2006.

HOLLNAGEL, E. Cognitive ergonomics: it's all in the mind. Ergonomics, v. 40, n. 10, p. 1170-82, 1997. 
HOLLNAGEL, E. Extended cognition and the future of ergonomics. Theoretical Issues in Ergonomics Science, v. 2, n. 3, p. 309-15, 2001.

HOOK, P. Domain Maps: Purposes, History, Parallels with Cartography, and Applications. 11th International Conference Information Visualization (IV'07). IEEE Computer Society Conference Publishing Services, 2007.

INGRAM, R.; BENFORD, S. Legibility Enhancement for Information Visualization. Proceedings of the 6th Conference on Visualization '95. IEEE Visualization. IEEE Computer Society, Washington DC., 1995.

JASTRZEBOWSKI, W.B. An outline of ergonomics or the science of work based upon the truths drawn from the science of nature (publicado en polaco en cuatro partes). Nature and Industry, v. 29 , p. $227-31$; v. 30, p. 236-44; v. 31, p. 244-51; v. 32, v. 33, p. 253-58, 1997.

JUAREZ, O.; HENDRICKSON, C.; GARRETT Jr., J.H. Evaluating Visualizations Based on the Performed Task. Proceedings of IEEE International Conference on Information Visualisation (IV'00),135-42, London, UK, 2000.

KARWOWSKI, W. Ergonomics and human factors: the paradigms for science, engineering, design, technology and management of human-compatible systems, Ergonomics, v. 48, n. 5, p. 436-63, 2005.

KEIM, D. A. Information Visualization and Visual Data Mining. IEEE Transactions on Visualization and Computer Graphics, v. 8, n. 1, 2002.

KEIM, D. A.; KRIEGEL, H. Visualization techniques for mining large databases: A comparison. IEEE Transactions on Knowledge and Data Engineering, v. 8, n. 6, p. 92338, 1996.

KOBSA, A. User Experiments with Tree Visualization Systems. Proceedings of the IEEE Symposium on Information Visualization (INFOVIS '04), 2004.

KOSARA, R. et al. Thoughts on User Studies: Why, How, and When? IEEE Computer Graphics \& Applications (CG\&A), Visualization Viewpoints, v. 23, n. 4, p. 20-5, Jul./Aug. 2003.

KOSHMAN, S. Comparing usability between a visualization and text-based system for information retrieval. Journal of Documentation, v. 60, n. 5, p. 565-80, 2004.

KOSHMAN, S. Visualization-based information retrieval on the Web. Library \& Information Science Research, v. 28, p. 192-207, 2006.

KRAIDY, U. Digital Media and Education: cognitive impact of information visualization. Learning, Media and Technology, v. 27, n. 3, p. 95-106, 2002.

LUZZARDI, R.G.; FREITAS, C.M.D.S. An Extended Set of Ergonomic Criteria for Information Visualization Techniques. Proceedings of the Seventh IASTED International Conference on Computer Graphics and Imaging (CGIM 2004), Kauai, Hawaii, 2004.

McCORMICK, D. H.; DeFANTI, T. A.; BROWN, M. D. Visualization in scientific computing. SIGGRAPH Computer Graphics Newsletter, v. 21, n. 6, 1987. 
NIGAY, L.; VERNIER, F. Design method of interaction techniques for large information spaces. Proceedings of the Working Conference on Advanced Visual interfaces (L'Aquila, Italy, May 24 - 27, 1998) T. Catarci, M. F. Costabile, G. Santucci, and L. Taranfino, Eds. AVI '98. ACM Press, New York, NY, 37-46, (1998).

NORMAN, D. A. Design principles for human-computer interfaces. Proceedings of $\mathrm{CHI} 83$, ACM., 1983.

PILLAT, R. M.; VALIATI, E. R. A.; FREITAS, C. M. D. S. Experimental Study on Evaluation of Multidimensional Information Visualization Techniques, Proceedings of the 2005 Latin American conference on Human-computer interaction CLIHC '05, ACM International Conference Proceeding Series, v. 124, p. 20-30, 2005.

PLAISANT, C. The Challenge of Information Visualization Evaluation. IEEE Proceedings of AVI 2004.

PLAISANT, C.; GROSJEAN, J.; BEDERSON, B. B. SpaceTree: supporting exploration in large node link tree, design evolution and empirical evaluation. Proceedings of the IEEE Symposium on Information Visualization 2002 (InfoVis '02), 2002.

ROBERTSON, G.; CARD, S. K.; MACKINLAY, J. D. The cognitive coprocessor architecture for interactive user interfaces. Proceedings of the 2nd Annual ACM SIGGRAPH Symposium on User interface Software and Technology UIST '89. ACM Press, New York, NY, p. 10-18, 1989.

SARAIYA; NORTH, C.; DUNCA, K. An Insight-based Methodology for Evaluating Bioinformatics Visualizations, IEEE Transactions on Visualization and Computer Graphics, v. 11, p. $443-56,2005$.

SARAIYA; NORTH, C.; DUNCA, K. An Evaluation of Microarray Visualization Tools for Biological Insight, Proceedings of the IEEE Symposium on Information Visualization (INFOVIS'04), Washington, D.C. v. 0, p. 1-8, 2004.

SHNEIDERMAN, B.; PLAISANT, C. Strategies for evaluating information visualization tools: multi-dimensional in-depth long-term case studies. Proceedings of the $2006 \mathrm{AVI}$ Workshop on Beyond Time and Errors: Novel Evaluation Methods For information Visualization BELIV '06. ACM Press, New York, NY, 1-7, 2006.

SMALL, H. Visualizing Science by Citation Mapping. Journal of the American Society for Information Science, v. 50, n. 9, p. 799-813, 1999.

STORRS, G. A. Conceptualisation of Human-Computer Interaction. IEEE Colloquium on Theory in Human-Computer Interaction (HCl), 4/1 - 4/5, 1989.

TAYLOR, F. W. The principles of scientific management. New York: Harper Bros., 1911, p. 5-29. Disponible en: http://melbecon.unimelb.edu.au/het/taylor/sciman.htm. Acesso em: 1 out. 2007.

TORY, M.; Möller, T. Evaluating visualizations: do expert reviews work? Computer Graphics and Applications, v. 25, n. 5, p. 8-11, 2005.

TORY, M.; Möller, T. Human Factors in Visualization Research. IEEE Transactions on Visualization and Computer Graphics, v. 10, n. 1, 2004. 
WANG, Y.; TEOH, S. T.; MA, K.-L. Evaluating the Effectiveness of Tree Visualization Systems for Knowledge Discovery. Proceedings of Eurographics/IEEE-VGTC Symposium on Visualization, p. 67-74, 2006.

WILSON, T. El modelado orientado al usuario: una perspectiva global. Anales de documentación, v. 2, p. 85-94, 1999.

WINCKLER, M. A.; PALANQUE, P.; FREITAS, C. Tasks and scenario-based evaluation of information visualization techniques. Proceedings of the 3rd Annual Conference on Task Models and Diagrams (Prague, Czech Republic, November 15 - 16, 2004. TAMODIA '04, ACM Press, New York, NY, v. 86, p. 165-172, 2004.

WISS, U; CARR, D.; JONSSON, H. Evaluating three-dimensional information visualization designs: a case study of three designs. Proceedings of the IEEE Conference on Information Visualization. London, UK, p.137-144, 1998.

ZUK, T.; CARPENDALE, M. S. T. Theoretical Analysis of Uncertainty Visualizations. Proceedings SPIE \& IS\&T Conf. Electronic Imaging, v. 60, p. 60, 2006: Visualization and Data Analysis 2006.

\title{
Title
}

Ergonomic influences on information visualization

\begin{abstract}
This paper aims to analyze and elucidate the interdisciplinary relationship between the Cognitive Ergonomics and Information Visualization, with the objective to assert the importance for the information professionals to consider ergonomics criteria for information visual interfaces design and evaluation. This historical and epistemological approach presented was interpreted and developed based on a qualitative analysis conducted with a set of information sources from scientific literature in both topics
\end{abstract}

\section{Keywords}

Cognitive Ergonomics - Information Visualization, Cognitive Ergonomics - Visual Interfaces.

\section{Título}

Influências ergonômicas na visualização da informação

\section{Resumo}

Este artigo pretende analisar e esclarecer algumas aproximações relativas à relação interdisciplinar que se estabelece entre a Ergonomia Cognitiva e a Visualização da Informação, na 
busca de um reconhecimento da necessidade de considerar critérios ergonômicos em sua estrutura e avaliação das interfaces visuais da informação pelos profissonais da informação. A interpretação e complementação do enfoque histórico e epistemológico se baseia na análise qualitativa de um conjunto de fontes de informação provenientes da literatura científica, relevante em ambos os campos.

\section{Palavras-chave}

Ergonomia Cognitiva - Visualização da Informação; Ergonomia Cognita - Interfaces Visuais.

Recebido em: 22.11.2009

Aceito em: 15.09.2010 